\title{
Closed Form Laurent-Puiseux Series of Algebraic Functions
}

\section{Wolfram Koepf}

Konrad-Zuse-Zentrum für Informationstechnik Berlin, Heilbronner Str. 10, D-10711 Berlin, Germany

Received December 9, 1993; revised version January 6, 1995

\begin{abstract}
There are several well-known algorithms to calculate the Puiseux series developments of the branches of an algebraic function. None of them, however, generates the series in closed form, even in those cases where such a formal result is available. They produce, instead, truncated series, and give information that can be used to handle the series as streams. Here we give a solution to the given problem. We combine an algorithm of D. V. and G. V. Chudnovsky that transforms the given algebraic equation into a differential equation for the function, and further into a recurrence equation for the Puiseux coefficients, with an algorithm of Koepf which in the case of hypergemetric type results in the formal series. A finite linear recurrence equation is optimal for a representation by streams. D. V. and G. V. Chudnovsky point out that their algorithm requires only $O(M)$ field operations if $M$ is the order of the number of series terms considered. However, from a practical point of view, it is of importance that the complexity of the resulting recurrence equation - as well as of the differential equation - can be extremely high, a fact, which we illustrate by an example. It turns out, that many algebraic functions of low order with a sparse representation are of hypergeometric type, and so closed form representations for the corresponding series can be given.
\end{abstract}

Keywords: formal power series, Laurent-Puiseux series, closed forms, hypergeometric terms and functions, functions of hypergeometric type, holonomic linear differential and recurrence equations.

\section{Introduction}

We consider algebraic functions $y(x)$ which are given by some bivariate polynomial equation

$$
F(x, y(x))=\sum_{k=0}^{N} p_{k}(x) y(x)^{k}=0
$$

with coefficient functions $p_{k} \in \mathbb{K}(x)$ where $\mathbb{K}$ is one of $\mathbb{Q}$, $\mathbb{R}$, or $\mathbb{C}$. 
Locally the branches of an algebraic function in a neighborhood of the origin $x=0$ can be represented by Laurent-Puiseux type series

$$
y(x)=\sum_{k \in Z} a_{k} x^{k} \quad\left(a_{-k_{0} / n} \neq 0, Z=\left\{-k_{0} / n,-\left(k_{0}+1\right) / n, \ldots\right\}\right)
$$

for some $k_{0} \in \mathbb{Z}$ and $n \in \mathbb{N}$, with coefficients $a_{k} \in \mathbb{C}(k \in Z)$. If there is a power series representation, i.e. if $k_{0} \geqq 0$ and $n=1$, the origin is called a regular, otherwise a singular point of the algebraic function.

There are well-known algorithms to find the coefficients of these representations iteratively, see e.g. [10]. Roughly, they consist of both a method to find initial values which, in general, are algebraic quantities, and of an iteration procedure to generate higher coefficients.

These algorithms are implemented in certain Computer Algebra systems, e.g. in Axiom [4] (previously Scratchpad), and Maple [3], see [11].

However, none of these algorithms leads to a formula for $a_{k}$. For that reason Axiom internally works with streams, and lazy evaluation, i.e. the series objects are given by a finite number of initial terms, and an (internally used) formula to calculate further coefficients, see e.g. [9]. Infinite series representations, however, are not supported.

We will not emphasize on the question how to find suitable initial values. However, we use an algorithm which generates a homogeneous linear recurrence equation for the coefficients searched for. In the special case of hypergeometric type, i.e. if the generated recurrence equation possesses only two summands, the closed form solution can explicitly be given. Many algebraic functions of low order are examples of that type.

\section{Algorithm to Generate of formal Laurent-Puiseux Expansion}

In [1]-[2] an algorithm was introduced generating a differential equation for (all branches of) $y(x)$, and a recurrence equation for its Laurent-Puiseux coefficients $a_{k}$. These authors gave an elegant algebraic argument that each algebraic function of $\operatorname{order} N$, given by (1), satisfies a homogeneous, linear differential equation

$$
\sum_{k=0}^{N} q_{k}(x) y^{(k)}(x)=0
$$

with polynomial coefficients $q_{k} \in \mathbb{K}(x)$ of order at most $N$ : Equation (3) states that the $N+1$ distinct functions $y^{(k)}(k=0, \ldots, N)$ are linearly dependent members of the (at most) $N$-dimensional linear space over $\mathbb{K}(x)$ which is generated by the extension of $\mathbb{K}(x)$ according to (1).

This algorithm can be combined with an algorithm that I developed in [5]. If the resulting recurrence equation of order $m$

$$
\sum_{j=0}^{m-1} P_{j} a_{k+j}=0 \quad\left(P_{0}, P_{m-1} \neq 0\right)
$$

contains only the two summands $j=0, m-1$, then we say that $y(x)$ is of hypergeometric type with symmetry number $m$, and an explicit formula for the coefficients can be found by the hypergeometric coefficient formula (see [5], Equation (2.2)), and some initial conditions. 
We implemented a version of this algorithm which is more on the lines of our development [5]-[7] (here especially the conversion of the differential equation to the recurrence equation is simpler, see [5], §6), and used it to calculate the Laurent-Puiseux expansions, and in particular the corresponding differential and recurrence equations, for many algebraic functions. The details of our implementation can be found in $[8]$.

It turns out that

1. many algebraic functions of low order, especially those with a sparse representation, are of hypergeometric type, and so closed form representations for the corresponding series can be given.

2. On the other hand, there are sparse low order algebraic functions for which the corresponding differential and recurrence equations have very high complexity.

In the following section we give examples illustrating the algorithm, and especially the issues mentioned.

\section{Examples}

First we use a simple algebraic function to illustrate the algorithm. We consider the algebraic equation

$$
y(x)^{2}+x^{2}-r^{2}=0
$$

i.e.

$$
y(x)^{2}=r^{2}-x^{2}
$$

Differentiation of (4) leads to

$$
2 x+2 y(x) y^{\prime}(x)=0
$$

with the explicit form

$$
y^{\prime}(x)=-\frac{x}{y(x)}
$$

The expression $y^{\prime}(x) / y(x)$ can be simplified using (5)

$$
\frac{y^{\prime}(x)}{y(x)}=-\frac{x}{y(x)^{2}}=-\frac{x}{r^{2}-x^{2}},
$$

which is independent of $y(x)$ so that the first order differential equation

$$
\left(r^{2}-x^{2}\right) y^{\prime}(x)+x y(x)=0
$$

holds for $y(x)$. By our general method (see [5], §6) the differential equation (8) is converted to the recurrence equation

$$
(k-1) a_{k}-(2+k) r^{2} a_{k+2}=0
$$

which is of hypergeometric type, and produces the two formal series

$$
y(x)= \pm \sum_{k=0}^{\infty} \frac{|r|(2 k) !}{4^{k} r^{2 k}(2 k-1) k !^{2}} x^{2 k} .
$$


Because the symmetry number of (9) is two, we need two initial values $y(0)$, and $y^{\prime}(0)$, which may be obtained by the usual Newton diagram method (see e.g. $[2], \S 7)$.

Next we consider the similar equation

$$
(y(x)-r)^{2}+x^{2}-r^{2}=0 .
$$

In this case a simplification of expression $y^{\prime}(x) / y(x)$ yields $y^{\prime}(x) / y(x)=x /\left(x^{2}-r y(x)\right)$ which is not independent of $y(x)$ so that no first order differential equation for $y(x)$ exists.

Thus one continues, and is finally led to the second order differential equation

$$
-r^{2} y^{\prime}(x)+r^{2} x y^{\prime \prime}(x)-x^{3} y^{\prime \prime}(x)=0 .
$$

Further we get the equivalent recurrence equation for the Laurent-Puiseux coefficients $a_{k}$

$$
(1-k) k a_{k}+k(2+k) r^{2} a_{k+2}=0
$$

which, again, is of hypergeometric type, producing the two formal series

$$
y(x)=\sum_{k=0}^{\infty} \frac{(2 k) !}{2 \cdot 4^{k} r^{2 k+1}(1+k) k !^{2}} x^{2+2 k},
$$

and

$$
y(x)=2 r-\sum_{k=0}^{\infty} \frac{(2 k) !}{2 \cdot 4^{k} r^{2 k+1}(1+k) k !^{2}} x^{2+2 k} .
$$

It turns out that algebraic functions of low degree (smaller than 5 or 6 ) with sparse representations often are of hypergeometric type. The following is a list of examples of that type which we generated with experimental implementations of our algorithm in Mathematica [12], and Maple [3]. Note that in our list, for brevity, we omit the argument $x$ of $y(x)$ :

$$
\begin{array}{ll}
F(x, y) & \text { recurrence equation } \\
x^{3}+x y-y^{2} & 2(k-1)(2 k-3) a_{k}+(k-1) k a_{k+1}=0, \\
x+y^{2}+x y^{3} & 27 k(1+k)(2+k) a_{k}+2(1+k)(4+k)(5+2 k) a_{k+3}=0, \\
x^{2}+y^{2}+x y^{3} & 3(1+k)(3 k-1)(7+3 k) a_{k}+4(1+k)(3+k)(5+k) a_{k+4}=0, \\
1+y^{2}+x y^{3} & 3(1+k)(1+3 k)(5+3 k) a_{k}+4(1+k)(2+k)(3+k) a_{k+2}=0 \\
-x+y+x y^{3} & 27 k(1+k) a_{k}+2(2+k)(7+2 k) a_{k+3}=0 \\
-y^{2}+x^{2} y^{4}-x & (1+4 k)(7+4 k) a_{k}+2(4+k)(5+2 k) a_{k+3}=0 \\
-1+x^{2} y^{2}+x y^{3} & 4(k-1)(1+k)(3+k) a_{k}-3(3+k)(5+3 k)(13+3 k) a_{k+4}=0, \\
-1+x^{2} y+x y^{2} & (k-1)(2+k) a_{k}+2(2+k)(7+2 k) a_{k+3}=0 \\
\left(x^{2}+y^{2}\right)^{3}-4 x^{2} y^{2} & 3(k-1)(3 k-1)(1+3 k) a_{k}-4 k(1+2 k)(3+2 k) a_{k+2}=0, \\
\left(x^{2}+y^{2}\right)^{4}-A x^{2} y^{2} & 256(1-k) k(2+k) a_{k}+3 A(1+k)(7+3 k)(11+3 k) a_{k+4}=0 \\
-y^{3}+x^{2} y^{4}-x^{2} & 256 k(2+k)(4+k)(6+k) a_{k} \\
& \quad+3(2+k)(10+k)(14+3 k)(22+3 k) a_{k+8}=0, \\
x^{2}+x y^{3}+y^{5} & 6 k(2 k-1)(3 k-1)(1+3 k) a_{k} \\
& \quad+5(5 k-1)(1+5 k)(2+5 k)(3+5 k) a_{k+1}=0 .
\end{array}
$$

We show that there exist low order algebraic functions that are not of the hypergeometric type considering the algebraic equation

$$
x y(x)^{2}-y(x)+1+x^{2}=0
$$


that corresponds to the recurrence equation

$$
\begin{aligned}
0= & 6(1+k)(2 k-1) a_{k}+4(3+k)(5+4 k) a_{k+2} \\
& -3(1+k)(4+k) a_{k+3}+2(5+k)(9+2 k) a_{k+4}-(5+k)(6+k) a_{k+5}
\end{aligned}
$$

of order 5 , which obviously is not of the hypergeometric type. Note that the algorithm generates the differential equation of lowest order, but not necessarily the recurrence equation of lowest order: In the present case the recurrence equation

$$
2(2 k-1) a_{k}+2(2 k+5) a_{k+2}-(k+4) a_{k+3}=0
$$

of order 3 is also valid. If we denote by $K a_{k}=a_{k+1}$ the shift operator with respect to $k$, then this corresponds to the fact that the operator polynomial corresponding to (13) has the (non-commutative) factorization

$$
\begin{aligned}
6(1+k)(2 k-1) & +4(3+k)(5+4 k) K^{2}-3(1+k)(4+k) K^{3} \\
& +2(5+k)(9+2 k) K^{4}-(5+k)(6+k) K^{5} \\
& =\left((k+5) K^{2}+3(k+1)\right)\left(2(2 k-1)+2(2 k+5) K^{2}-(k+4) K^{3}\right) .
\end{aligned}
$$

As another sparse example of low order we consider the equation of fifth order (see e.g. [11], Example 1)

$$
y(x)^{5}+2 x y(x)^{4}-x y(x)^{2}-2 x^{2} y(x)+x^{4}-x^{3}=0 .
$$

It turns out that no differential equation of order smaller than 5 exists. The differential equation of order five that is valid for $y(x)$ has the following complexity:

1. the coefficients of each expression $y^{(k)}(k=0, \ldots, 5)$ are polynomials of degree 31 ,

2. those polynomials are nonsparse with coefficients that are typically 15-digit integers,

3. expanding the differential equation yields 190 summands.

The final recurrence equation has the following properties:

1. It is of order 32 ,

2. the coefficients of each expression $a_{k+j}(j=0, \ldots, 31)$ are polynomials of degree 5 ,

3. those polynomials are nonsparse with coefficients that are typically 20 -digit integers with maximum 11235463054441863110400 ,

4. expanding the recurrence equation yields 192 summands.

This example shows that even for small degree the complexity of the resulting differential and recurrence equations can be extremely high, and moreover that in order to use the recurrence equation many initial values may be needed.

D. V. and G. V. Chudnovsky point out that their algorithm requires only $O(M)$ field operations if $M$ is the order of the number of series terms considered. The above example shows, however, that in practical situations the computing overhead given by the conversion of $y(x)$ into the corresponding (nonsparse) recurrence equation cannot be neglected even for sparsely given algebraic functions, and the computing time and memory requirements can be tremendous even for small $M$ despite the given complexity order. 


\section{References}

1. Chudnovsky, D. V., Chudnovsky, G. V.: On expansion of algebraic functions in power and Puiseux series I. J. Complexity 2, 271-294 (1986)

2. Chudnovsky, D. V., Chudnovsky, G. V.: On expansion of algebraic functions in power and Puiseux series II. J. Complexity 3, 1-25 (1987)

3. Char, B. W., Geddes, K. O., Gonnet, G. H., Leong, B. L., Monagan, M. B., Watt, S. M.: Maple V. Language Reference Manual. Berlin, Heidelberg, New York: Springer 1991

4. Jenks, R. D., Sutor, R. S.: Axiom. Berlin, Heidelberg, New York: Springer 1993

5. Koepf, W.: Power series in Computer Algebra. J. Symb. Comp. 13, 581-603 (1992)

6. Koepf, W: Algorithmic development of power series. In: Artificial intelligence and symbolic mathematical computing. Calmet, J., Campbell, J. A. (eds) International Conference AISMC-1, Karlsruhe, Germany, August 1992, Proceedings. Lecture Notes in Computer Science vol. 737. Berlin, Heidelberg, New York: Springer (1993), pp 195-213

7. Koepf, W:: Examples for the algorithmic calculation of formal Puiseux, Laurent and power series. SIGSAM Bull. 27, 20-32 (1993)

8. Koepf, W: A new algorithm for the development of algebraic functions in Puiseux series. Preprint B-92-21 Fachbereich Mathematik der Freien Universität Berlin, 1992

9. Norman, A. C.: Computing with formal power series. Transactions on mathematical software 1, ACM Press: New York, 346-356(1975)

10. Wall, B.: Puiseux expansion. An annoted bibliography. Technical report, RISC-Linz Report Series No. 91-46.1, 1991

11. Wall, B.: On implementations of Puiseux series expansion in a Computer Algebra System. RISC-Linz Report Series No. 92-40, 1992

12. Wolfram, St.: Mathematica. A system for doing mathematics by Computer. Addison-Wesley Publ. Comp., Redwood City, CA, 1991 